Alice M. Balderacchi, Valentina Barzon, Stefania Ottaviani, Alessandra Corino, Michele Zorzetto, Marion Wencker, Angelo G. Corsico and Ilaria Ferrarotti*

\title{
Comparison of different algorithms in laboratory diagnosis of alpha1-antitrypsin deficiency
}

https://doi.org/10.1515/cclm-2020-1881

Received December 29, 2020; accepted February 17, 2021;

published online March 5, 2021

\section{Abstract}

Objectives: Alpha1-antitrypsin deficiency (AATD) is an inherited condition that predisposes individuals to an increased risk of developing lung and liver disease. Even though AATD is one of the most widespread inherited diseases in Caucasian populations, only a minority of affected individuals has been detected. Whereas methods have been validated for AATD testing, there is no universally-established algorithm for the detection and diagnosis of the disorder. In order to compare different methods for diagnosing AATD, we carried out a systematic review of the literature on AATD diagnostic algorithms.

Methods: Complete biochemical and molecular analyses of 5,352 samples processed in our laboratory were retrospectively studied using each of the selected algorithms.

Results: When applying the diagnostic algorithms to the same samples, the frequency of False Negatives varied from 1.94 to $12.9 \%$, the frequency of True Negatives was $62.91 \%$ for each algorithm and the frequency of True Positives ranged from 24.19 to $35.15 \%$. We, therefore, highlighted some differences among Negative Predictive Values, ranging from 0.83 to 0.97 . Accordingly, the sensitivity of each algorithm

Alice M. Balderacchi and Valentina Barzon contributed equally to this work.

*Corresponding author: Ilaria Ferrarotti, BS PhD, Centre for Diagnosis of Inherited Alpha-1 Antitrypsin Deficiency, Laboratory of Biochemistry and Genetics, Institute for Respiratory Disease, Department of Internal Medicine and Therapeutics, University of Pavia, Fondazione IRCCS Policlinico San Matteo, V.le Golgi 19, 27100 Pavia, Italy, Phone: +390382 502620,

E-mail: ilaria.ferrarotti@unipv.it. https://orcid.org/0000-00034892-4192

Alice M. Balderacchi, Valentina Barzon, Stefania Ottaviani, Alessandra Corino, Michele Zorzetto and Angelo G. Corsico, Centre for Diagnosis of Inherited Alpha-1 Antitrypsin Deficiency, Laboratory of Biochemistry and Genetics, Institute for Respiratory Disease, Department of Internal Medicine and Therapeutics, University of Pavia, Fondazione IRCCS Policlinico San Matteo, Pavia, Italy Marion Wencker, Conresp, Loerzweiler, Germany ranged between 0.61 and 0.95 . We also postulated $1.108 \mathrm{~g} / \mathrm{L}$ as optimal AAT cut-off value, in absence of inflammatory status, which points to the possible presence of genetic AATD.

Conclusions: The choice of the diagnostic algorithm has a significant impact on the correct diagnosis of AATD, which is essential for appropriate treatment and medical care. The fairly large number of possible false negative diagnoses revealed by the present paper should also warn clinicians of negative results in patients with clinically-suspected AATD.

Keywords: algorithm; alpha1-antitrypin; cut-off; diagnosis; genetic test; laboratory analysis; rare diseases.

\section{Introduction}

Alpha1-antitrypsin deficiency (AATD) is an inherited condition that predisposes individuals to an increased risk of developing lung and liver disease even at an early age.

Alpha1-antitrypsin (AAT) protein is encoded by the protease inhibitor (PI) locus, located on the long arm of chromosome 14 (14q31-32.3). The PI gene (also known as SERPINA1) spans $12.2 \mathrm{~kb}$ and is organized into four coding (II, III, IV and V) and three non-coding (Ia, Ib and Ic) exons. The encoded protein includes 394 aminoacids with the active site of the enzyme inhibitor located at methionine 358 [1].

The SERPINA1 gene is highly polymorphic with more than 120 known variants [2].

For clinical purposes, allelic variants of AAT have been classified into three main categories [3]: normal, referred to as M (M1, M2, M3, M4) and characterized by AAT plasma levels within reference ranges in the general population; deficient, mostly characterized by missense mutations, including the common PI^Z (Glu342Lys) and PIS (Glu264Val) mutations or small deletions, such as the $M_{\text {malton }}$ variant (p.Phe52del) associated with decreased, but still detectable AAT plasma levels; null, currently designated Q0, resulting from nonsense or frameshift mutations leading to premature stop codons, with no detectable AAT plasma levels and associated with an increased risk of developing emphysema [4]. To date, more than 130 alleles have been described in the literature, most of 
which are associated with a significant decrease in the level of circulating AAT (deficiency variants) or lead to a total absence of the protein (null alleles).

Even if AATD is one of the most widespread inherited diseases in Caucasian populations, identifying and diagnosing affected patients is still unsatisfactory, with only a minority of affected individuals being detected, long delays between initial symptoms and diagnosis and evidence suggesting that AATD subjects with indicative symptoms are likely to see many physicians before an initial diagnosis is made [5].

Laboratory diagnosis of AATD has evolved over the last 55 years, since the first cases of the disorder were reported, based on paper electrophoresis, which recognized different protein variants of AAT according to the position and appearance of the precipitation pattern (normal or slow) [6]. These methodological advances have facilitated the more widespread application of rapid, convenient and cost-effective AATD tests, leading to an increase in the number of individuals diagnosed with the disorder.

Laboratory diagnosis of AATD currently consists of serum biochemical analyses to evaluate protein deficiency and electrophoretic abnormalities, as well as genetic analysis to identify gene variants responsible for the protein deficiency [7].

A large number of mutations have been identified in the coding regions of the SERPINA1 gene. However, extending sequence analysis to some intronic portions has allowed the detection of very rare mutations such as $\mathrm{Q} 0_{\text {porto }}$ [8], $\mathrm{Q}_{\text {madrid }}$

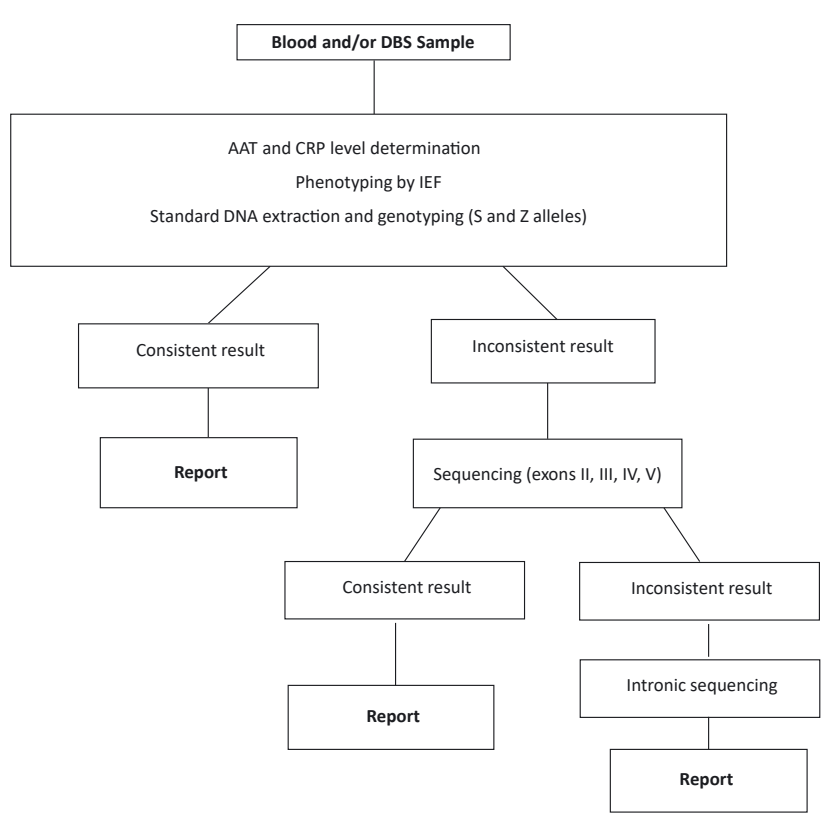

Figure 1: General decision tree related to the algorithm \#7 used in the current cohort.
[9] and $\mathrm{M}_{\text {whitstable }}[10]$, which lead to an increased risk of developing lung disease.

In order to further increase the efficiency of AATD testing, each specialized laboratory has developed and evaluated its own diagnostic algorithm using different biological materials, i.e. blood, plasma, dried blood spots (DBS), buccal swabs, relating to the epidemiology of AATD and the occurrence of mutations in their countries. While methods have been validated for AATD testing, there is no universally-established algorithm for the detection and diagnosis of the disorder [11].

Thus, we decided to compare different existing practices and evaluate how the diagnostic algorithm might affect AATD diagnosis. We also postulate optimal AAT cutoff values, according to inflammatory status, which point to the possible presence of genetic AATD.

\section{Materials and methods}

\section{Literature search}

In order to compare different methods for diagnosing AATD, we carried out a systematic review of the literature on AATD diagnostic algorithms using the following electronic databases: PUBMED, MEDLINE (Ovid) and MEDLINE In-Process (Ovid).

Publications were extracted from these databases using key search terms and any possible combinations using the logical operator AND. Key search terms included "AATD" AND "Diagnosis" AND "Algorithm", and "AATD" AND "Laboratory". We also studied nonautomatic reference lists in related publications to discover additional studies. Any article deemed to be eligible was thoroughly evaluated before being included in the study. Only papers published in English were taken into consideration.

\section{Establishing a reference algorithm}

We developed a diagnostic algorithm using a combined approach consisting of quantitative tests to evaluate AAT blood concentration and qualitative tests to identify all mutated alleles causing AATD (Figure 1). The diagnostic process was performed entirely on blood and/or DBSs.

AAT and C-reactive protein (CRP) concentrations in blood and DBSs were measured using nephelometry to measure the intensity of scattered light which is directly related to the concentration of the dispersed phase $[12,13]$. We determined both AAT and CRP using Array 360 System (Beckmann Coulter S.P.A., Milan, Italy).

To identify the mutated allele(s) producing a major structural change in the protein and deficiency in the AAT level, we performed different qualitative tests. We performed Isoelectric focusing (IEF) on ready-to-use agarose gels, using the semi-automatic Sebia Hydrasys ${ }^{\circledR}$ System (Sebia, Evry, France) and the Hydragel 18 A1AT Isofocusing ${ }^{\circledR}$ kit to detect AAT bands corresponding to the different variants [14, 15]. All samples were also genotyped to evaluate the presence of the two most common deficiency variants, $Z$ (p.E366K c.1096G $>A$ rs17580) and $S$ (p.E288V c.863A>T rs28929474). Our method for S/Z genotyping was 
based on DNA extracted from blood or DBSs and typed by PCR with fluorescently-labelled Taq-Man probes (Vic or Fam labels) on a LigthCycler 480 (Roche Diagnostics) [16].

We estimated the value of $1.2 \mathrm{~g} / \mathrm{L}$ of AAT serum concentration (with normal CRP values) as the decisional value to improve qualitative analysis by sequencing, in case of negative genotyping (not $\mathrm{S}$ not $Z$ ). Therefore, in the event of serum level below decisional value and/or discrepancies between genotype or phenotype, we used the Sanger method to sequence the SERPINA1 gene, allowing us to precisely identify AAT alleles [11].

In addition, intronic sequencing proved to be effective in improving molecular diagnosis and correctly identifying hereditary AATD by detecting extremely rare mutations which may cause reduced AAT protein production [7].

The algorithm was applied to a cohort of 5.352 samples sent to the Centre for the Diagnosis of Inherited Alpha-1 Antitrypsin Deficiency by Italian clinicians in the period spanning January 2013-February 2018. Samples which could not be processed due to poor sampling or storage before diagnosis were not included in the cohort. Based on the location of the working hospitals or outpatient clinics of the physicians who sent the samples, regional samples were as follows: 51.7, 22.0 and $26.3 \%$ from Northern, Central and Southern Italy, respectively. Most samples were from subjects born in Italy; 171 out of 5.352 subjects (3.2\%) were born outside Italy. Among them, we had a prevalence of male (59\%). Mean age of the whole cohort was 53.21 years.

All biochemical and genetic analyses performed on our cohort of subjects with suspected AATD enabled us to obtain a robust final diagnosis

\section{Retrospective testing and receiver operating characteristic (ROC) curve}

Complete biochemical and molecular analyses of 5.352 samples processed in our laboratory were retrospectively studied for the selected algorithms.

Considering the partial results of our algorithm, such as AAT concentration, phenotyping, genotyping, and sequencing, we applied the selected algorithms in Table 1 to each sample to obtain the final diagnoses. By comparing these results with the final genotype obtained at our centre, we were able to calculate the percentage of False Negatives (FN), True Negatives (TN) and True Positives (TP) in each algorithm and consequently determine the sensitivities of each algorithm. We determined the Negative Predictive Value (NPV) of all the algorithms to establish whether they were capable of providing an accurate diagnosis.

The optimal threshold for AAT serum levels was determined by plotting a receiver operating curve (ROC), a graphical technique for assessing the test's capacity to discriminate between those with and without disease [23]. To this end, all samples with at least one pathological SERPINA1 allele were taken to be positive. The accuracy of diagnostic tests was confirmed by their sensitivity according to the choice of cut-off value.

\section{Results}

The different algorithms selected for AATD diagnosis published in international journals are shown in Table 1.
This table also compares biochemical and genetic analyses of selected algorithms, as well as the diagnostic approach in the cohort of samples we used as a reference. Since algorithm \#7 was the most complete in comparison to others, we applied it to our cohort of 5.352 samples and took the derived diagnosis as a reference value to evaluate the sensitivity of previously published algorithms. 2.047 samples (38.2\%) in our cohort were positive, i.e. with at least one pathological SERPINA1 allele.

The comparison of each algorithm applied to our cohort of 5.352 samples allowed us to calculate the rate of FN, TN, $\mathrm{TP}$, highlighting considerable variations and emphasising different rates of False Negatives (Figure 2).

The present comparison thus enables us to assess false positive samples, since we assumed that positive diagnoses were correct.

When applying the diagnostic algorithms to the same samples, the frequency of False Negatives varied from 1.94 to $12.9 \%$, the frequency of $\mathrm{TN}$ was $62.91 \%$ for each algorithm and the frequency of TP ranged from 24.19 to $35.15 \%$. We, therefore, highlighted some differences among NPV, as shown in Table 2, ranging from 0.83 to 0.97. Accordingly, the sensitivity of each algorithm ranged between 0.61 and 0.95 .

Overall, 71 samples out of 5.323 (1.3\%) were FN in all selected algorithms. Of these, 12 samples had normal AAT and above normal CRP values and reported pathological ( ${ }^{\star}{ }^{\star} \mathrm{MM}_{\text {wurzburg, }}$ p.P393S rs28931570, five samples), likelypathological $\left(\mathrm{PI}{ }^{\star} \mathrm{M} / \mathrm{S}_{\text {munich}}, \quad \mathrm{p} . \mathrm{S} 354 \mathrm{~F}-\mathrm{rs} 201788603\right.$, one sample) genotypes and new pathological variants (6 samples), whose molecular mechanisms are still being investigated. Moreover, 56 samples had normal CRP values and AAT was slightly above the cut-off value. These included 15 $\mathrm{PI}^{\star} \mathrm{M} / \mathrm{M}_{\text {wurzburg }}, 8 \mathrm{PI}^{\star} \mathrm{M} / \mathrm{S}_{\text {munich }}, 1 \mathrm{PI}{ }^{\star} \mathrm{M} / \mathrm{M}_{\text {varallo }}$ [24], $2 \mathrm{PI}^{\star} \mathrm{M} /$ $\mathrm{QO}_{\text {pordenone }}$ (c.1052delT rs7255374), $1 \mathrm{PI}^{\star} \mathrm{M} / \mathrm{QO}_{\text {porto }}$ [9], 1

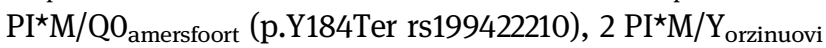
(p.P415H [25]), 2 PI^M/X $\mathrm{X}_{\text {chistchurch }}$ (p.E387K rs121912712) and 24 samples bearing one novel allele (23 in combination with $M$ alleles, one in combination with the $Z$ allele) which can be considered pathological after applying the pathogenicity prediction [2].

Finally, four samples had normal CRP values, and AAT $\leq 1.2 \mathrm{~g} / \mathrm{L}$ (namely $2 \mathrm{PI}^{\star} \mathrm{M} / \mathrm{M}_{\text {wurzburg }}$ and $2 \mathrm{PI}^{\star} \mathrm{M} / \mathrm{M}_{\text {whitstable }}$ [10]).

Some algorithms were less sensitive than others. In particular, algorithm \#4, with a sensitivity of 0.65 failed to detect positivity in 210 samples (namely $105 \mathrm{PI}^{\star} \mathrm{MZ}, 101$ $\mathrm{PI}^{\star} \mathrm{MS}, 2 \mathrm{PI}^{\star} \mathrm{SS}, 2 \mathrm{PI}^{\star} \mathrm{SZ}$ ) that were correctly detected by other algorithms. Algorithms \#1 and \#5, with a sensitivity of 0.76, provided 155 False Negative samples that were accurately detected by the other algorithms. 


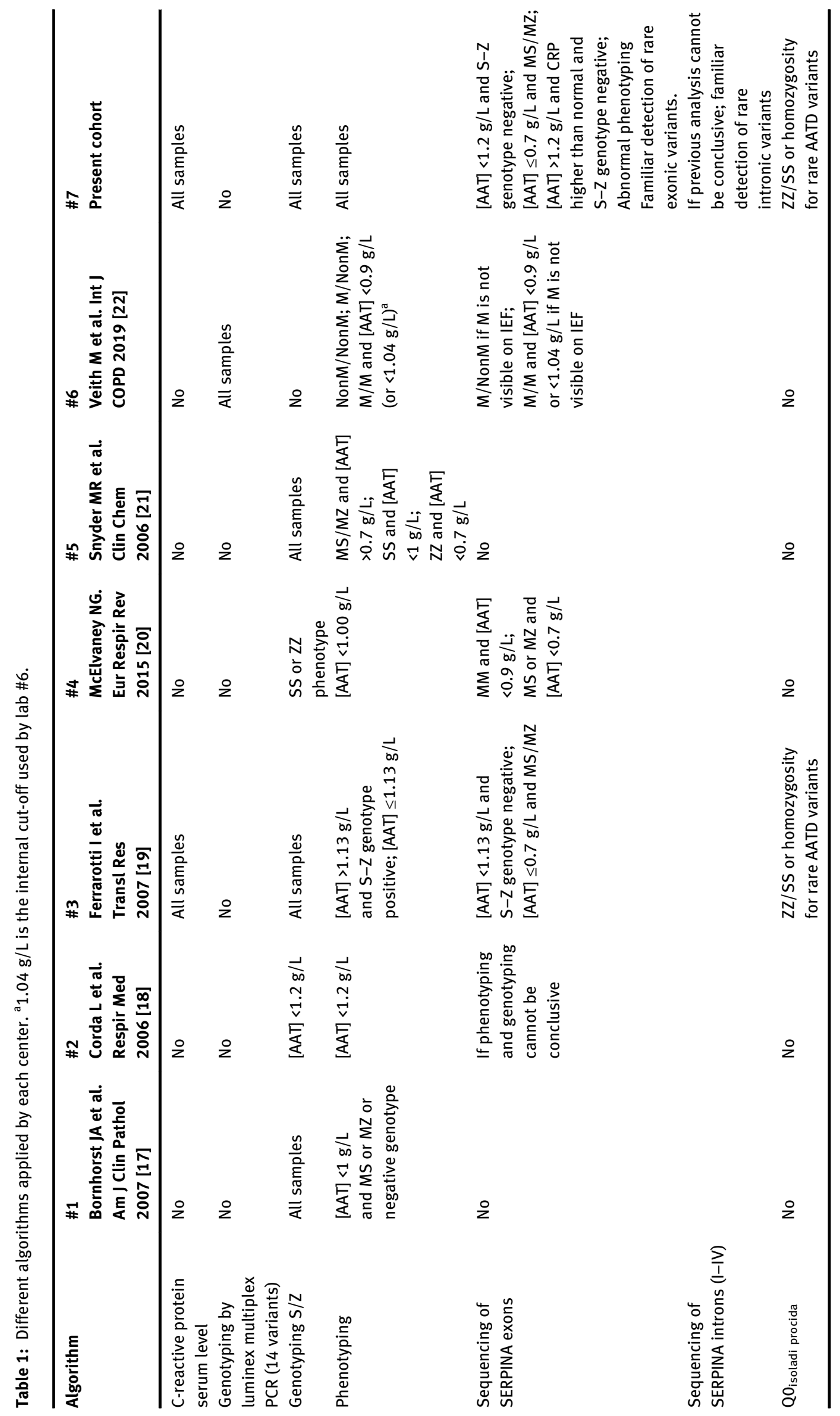




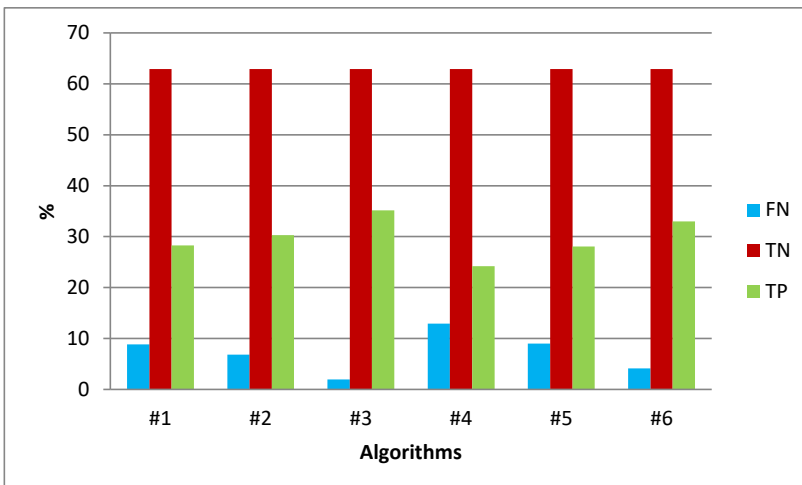

\begin{tabular}{|c|c|c|c|c|c|c|}
\hline & $\# 1$ & $\# 2$ & $\# 3$ & $\# 4$ & $\# 5$ & $\# 6$ \\
\hline$\square$ FN & 8.84 & 6.83 & 1.94 & 12.9 & 9.02 & 4.11 \\
\hline$\square T N$ & 62.91 & 62.91 & 62.91 & 62.91 & 62.91 & 62.91 \\
\hline$\square$ TP & 28.25 & 30.26 & 35.15 & 24.19 & 28.07 & 32.97 \\
\hline
\end{tabular}

Figure 2: Percentage of False Negatives, True Negatives and True Positives of each algorithm.

Table 2: Percentage of False Negative and Negative Predictive Values of each algorithm.

\begin{tabular}{llr}
\hline Algorithm & NPV & Sensitivity \\
\hline$\# 1$ & 0.88 & 0.76 \\
$\# 2$ & 0.90 & 0.82 \\
$\# 3$ & 0.97 & 0.95 \\
$\# 4$ & 0.83 & 0.65 \\
$\# 5$ & 0.87 & 0.76 \\
$\# 6$ & 0.93 & 0.89 \\
\hline
\end{tabular}

We used the ROC curve to test all samples; namely 4.550 with normal CRP values and 802 with $\mathrm{CRP} \geq 0.008 \mathrm{~g} / \mathrm{L}$, using a cut-off value of $0.008 \mathrm{~g} / \mathrm{L}$.

The ROC curve shown in Figure 3A identified $1.108 \mathrm{~g} / \mathrm{L}$ as the optimal cut-off value for suspected genetic AAT deficiency (78.3\% sensitivity and $86.4 \%$ specificity) in patients in the non-acute phase, as indicated by $\mathrm{CRP}<0.008 \mathrm{~g} / \mathrm{L}$.

The second ROC curve (Figure 3B) highlights $1.56 \mathrm{~g} / \mathrm{L}$ as the optimal cut-off value (82.9\% sensitivity and $76.5 \%$ specificity) in patients with CRP $>0.008 \mathrm{~g} / \mathrm{L}$ and likely inflammatory status.

\section{Discussion}

AATD is a genetic disorder frequently under- or misdiagnosed. Its diagnosis consists of biochemical serum analyses to evaluate protein deficiency and electrophoretic abnormalities as well as genetic analysis to identify SERPINA1 gene variants, responsible for the protein deficiency [7]. An accurate and complete identification of the AATD
A

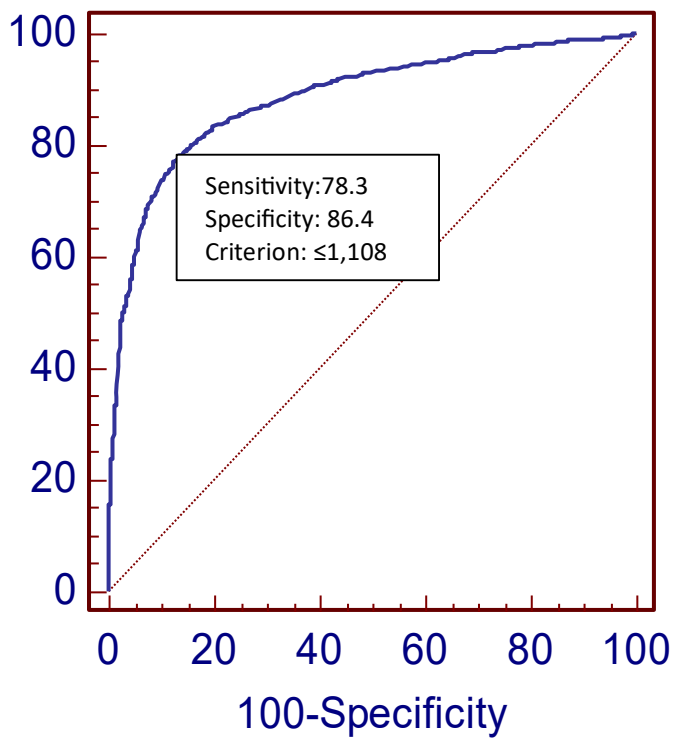

B

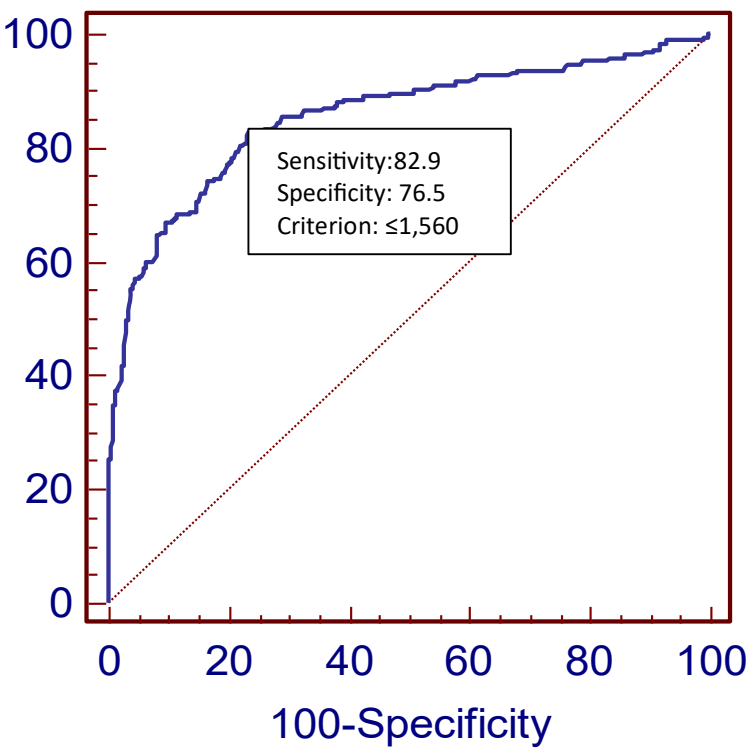

Figure 3: ROC curve in patients with normal CRP (A) and with CRP>0.008 g/L (B).

geno/phenotype is clinically fundamental when deciding on potential treatment options for individual patients, including augmentation therapy [26]. Further to this, the precise and early diagnosis of severe and intermediate AATD allows preventive measures to be taken, the most 
important of which is the avoidance of exposure to environmental pollutants and smoke (including the inhalation of second-hand smoke). Therefore, the role of laboratory tests in the diagnosis of AAT is crucial. Notwithstanding this, there is no universally-established algorithm for the detection and diagnosis of the disorder used by laboratories and countries. Currently, these algorithms depend on different criteria such as efficient diagnostic facilities and expertise, occurrence of mutations, country-based indications and availability of augmentation therapy. In the present paper, a retrospective application of different algorithms to the same cohort of samples aimed to evaluate the impact of the choice of diagnostic approach on the final AATD diagnosis. Importantly, we wish to emphasise that our use of the term 'diagnostic algorithm' refers to a structured diagnostic approach and not to a single diagnostic test, given the recent European Respiratory Society (ERS) statement that clearly supports the roles of Reference Centres in the diagnosis and management of rare diseases such as AATD [26].

The comparison of selected algorithms by our systematic review [17-22] highlights glaring differences between diagnostic approaches. First and foremost, most algorithms did not use a clinical indicator of inflammation such as C-reactive protein. The concentration of AAT is genetically determined. However, several studies have noted that inflammation, as indicated by CRP, can significantly affect observed AAT levels in genotypes associated with intermediate deficiency [13, 16, 27-29]. Therefore, lack of evidence concerning the acute phase stage in diagnostic processes could be a source of false negative results, especially in those algorithms where detected AAT concentrations below established cut-off values are mandatory for further tests $[18,20]$. Another important difference regards whether or not SERPINA1 sequencing should be performed to detect rare variants. Following the publication of a pioneering Italian study, the presence of rare pathological variants has proven that they are not confined to single countries, as previously thought [30]. To date, many missense [2] and Null [31] mutations of the SERPINA1 gene are known. The identification of the exact molecular mechanisms underlying AATD undoubtedly helps in defining different diagnostic risk levels for lung and liver disease displayed by varying AATD genotypes. The accuracy of results varies according to the detection sensitivity of rare variants in the algorithms. This is the main cause of a reduced sensitivity to 0.76 for algorithms that are unable to detect rare pathological variants $[17,21]$.

In Table 2, only two out of six algorithms displayed a $\mathrm{NPV}>0.90$, which is relatively acceptable for the diagnosis of a rare disease. Interestingly, the higher sensitivity was reached by diagnostic algorithm \#3 [19] including CRP testing and using a higher decisional value of AAT concentration to move to molecular tests. This diagnostic algorithm was used by our group until seven years ago, when it was updated with a higher cut-off value (1.2 instead of $1.13 \mathrm{~g} / \mathrm{L}$ ) and the inclusion of intronic sequencing. These improvements led to an increased diagnostic accuracy.

Finally, the ROC curve highlights two main points. The former is the salient role of CRP evaluation as a marker of inflammation in the quantitative evaluation of AAT. The latter is the correct application of cut-off values in diagnosing AATD in Reference Centres. If we limited qualitative diagnosis to samples with an AAT concentration below cutoff values $(1.108 \mathrm{~g} / \mathrm{L})$, we would not reach a high sensitivity rate (0.78). On the contrary, if the cut-off value is used in screening steps before referring samples to Diagnostic Reference Centres, it could be a useful instrument to target diagnosis.

\section{Conclusions}

The present paper is unable to estimate the cost-effective relationship of each algorithm. Clearly, further tests are needed to establish the high accuracy of the results. Current "precision medicine" is providing additional, innovative dimensions to the concept of diagnosis [32], since a timely and accurate diagnosis is one of the main goals of patients with AATD [33] and rare diseases [34].

We conclude that the choice of the diagnostic algorithm has a significant impact on the correct diagnosis of AATD, which is essential for appropriate treatment and medical care. We also underline the importance of CRP evaluation, as acute phase protein marker, to reduce the false negative results. The fairly large number of possible false negative diagnoses revealed by the present paper should also warn clinicians of negative results in patients with clinically-suspected AATD. In these cases, re-testing with more sensitive algorithms could help to achieve an accurate diagnosis of AATD.

Acknowledgment: All authors have read the journal's authorship statement and policy on disclosure of potential conflicts of interest.

Research funding: No specific funds were used to produce this manuscript

Author contributions: All authors have accepted responsibility for the entire content of this manuscript and approved its submission. I.F., M.W. and A.G.C conceived the 
paper, I.F. and S.O. designed the experiments; A.M.B., V.B., A.C. and S.O. performed the experimental part; I.F. and S.O. reviewed the literature; A.M.B. and V.B. analysed the results; A.M.B. and V.B. performed retrospective analysis; A.M.B., V.B. and I.F. wrote the paper; A.G.C., M.W. and M.Z. critically revised the paper. All the authors approved the final version of the manuscript.

Competing interests: The authors declare that they have no competing interest.

Informed consent: It is a retrospective analysis without clinical and personal data, therefore consent to participate is not applicable.

Ethics approval and consent to participate: It is a retrospective analysis without clinical and personal data, therefore consent to participate is not applicable.

\section{References}

1. Stoller JK, Aboussouan LS. Alpha1-antitrypsin deficiency. Lancet 2005;365:2225-36.

2. Giacopuzzi E, Laffranchi M, Berardelli R, Ravasio V, Ferrarotti I, Gooptu B, et al. Real-world clinical applicability of pathogenicity predictors assessed on SERPINA1 mutations in alpha1-antitrypsin deficiency. Hum Mutat 2018;39:1203-13.

3. American Thoracic Society, European Respiratory Society. American Thoracic Society/European Respiratory Society statement: standards for the diagnosis and management of individuals with alpha1-antitrypsin deficiency. Am J Respir Crit Care Med 2003;168:818-900.

4. Popławska B, Janciauskiene S, Chorostowska-Wynimko J. Genetic variants of alpha-1 antitrypsin: classification and clinical implications. Pneumonol Alergol Pol 2013;81:45-54.

5. Köhnlein T, Janciauskiene S, Welte T. Diagnostic delay and clinical modifiers in alpha-1 antitrypsin deficiency. Ther Adv Respir Dis 2010;4:279-87.

6. Eriksson $S$, Laurell CB. A new abnormal serum globulin a1-antitrypsin. Acta Chem Scad 1963;17:150-3.

7. Ferrarotti I, Ottaviani S. Laboratory diagnosis. In: Strnad P, Brantly ML, Bals R, editors. $\alpha 1$-Antitrypsin deficiency (ERS monograph). Sheffield: European Respiratory Society; 2019: 39-51pp.

8. Seixas S, Mendonça C, Costa F, Rocha J. Alpha1-antitrypsin null alleles: evidence for the recurrence of the L353fsX376 mutation and a novel $\mathrm{G}->\mathrm{A}$ transition in position +1 of intron IC affecting normal mRNA splicing. Clin Genet 2002;62:175-80.

9. Lara B, Martínez MA, Blanco I, Ferrarotti I, Rodrìguez Frìas F, Velasco $E$, et al. Molecular characterization of the new alpha-1 antitrypsin deficiency null allele $\mathrm{Pi}^{\star} \mathrm{QO}_{\text {Madrid. }}$. Respir Res 2014;15:125.

10. Ambrose HJ, Chambers SM, Mieli-Vergani G, Ferrie R, Newton CR, Robertson NH. Molecular characterization of a new alpha1-antitrypsin $M$ variant allele, $M_{\text {whitstable: implications for }}$ DNA-based diagnosis. Diagn Mol Pathol 1999;8:205-10.

11. Miravitlles M, Herr C, Ferrarotti I, Jardi R, Rodrìguez Frìas F, Luisetti $M$, et al. Laboratory testing of individuals with severe $\alpha 1$-antitrypsin deficiency in three European centres. Eur Respir J 2010;35:960-8.

12. Gorrini M, Ferrarotti I, Lupi A, Bosoni T, Mazzola P, Scabini R, et al. Validation of a rapid, simple method to measure

alpha1-antitrypsin in human dried blood spots. Clin Chem 2006; 52:899-901.

13. Ottaviani S, Gorrini M, Scabini R, Kadija Z, Paracchini E, Mariani F, et al. $C$ reactive protein and alpha1-antitrypsin: relationship between levels and gene variants. Transl Res 2011;157:332-8.

14. Zerimech F, Hennache G, Bellon F, Barouh G, Jaques Lafitte J, Porchet N, et al. Evaluation of a new Sebia isoelectrofocusing kit for alpha 1-antitrypsin phenotyping with the Hydrasys System. Clin Chem Lab Med 2008;46:260-3.

15. Ottaviani S, Barzon V, Balderacchi AM, Corsico AG, Ferrarotti I. Improvement of the phenotyping detection of the rare alpha1-antitrypsin deficiency $M_{\text {wurzburg }}$ variant. In: ERS International Congress 2019.

16. Ferrarotti I, Thun GA, Zorzetto M, Ottaviani S, Imboden M, Schindler $C$, et al. Serum levels and genotype distribution of $\alpha 1$-antitrypsin in the general population. Thorax 2012;67: 669-74.

17. Bornhorst JA, Procter M, Meadows C, Ashwood ER, Mao R. Evaluation of an integrative diagnostic algorithm for the identification of people at risk for alpha1-antitrypsin deficiency. Am J Clin Pathol 2007;128:482-90.

18. Corda L, Bertella E, Pini L, Pezzini A, Medicina D, Boni E, et al. Diagnostic flow chart for targeted detection of alpha1-antitrypsin deficiency. Respir Med 2006;100:463-70.

19. Ferrarotti I, Scabini R, Campo I, Ottaviani S, Zorzetto M, Gorrini M, et al. Laboratory diagnosis of alpha1-antitrypsin deficiency. Transl Res 2007;150:267-74.

20. McElvaney NG. Diagnosing $\alpha 1$-antitrypsin deficiency: how to improve the current algorithm. Eur Respir Rev 2015;24:52-7.

21. Snyder MR, Katzmann JA, Butz ML, Wiley C, Yang P, Dawson DB, et al. Diagnosis of alpha-1-antitrypsin deficiency: an algorithm of quantification, genotyping, and phenotyping. Clin Chem 2006; 52:2236-42.

22. Veith M, Klemmer A, Anton I, El Hamss R, Rapun N, Janciauskiene $S$, et al. Diagnosing alpha-1-antitrypsin deficiency using a PCR/luminescence-based technology. Int J Chronic Obstr Pulm Dis 2019.

23. Altman DG, Bland JM. Diagnostic tests 3: receiver operating characteristic plots. BMJ 1994;309:188.

24. Coni P, Pili E, Convertino G, Pichiri G, Balestrino A, Del Mastro M, et al. $M_{V \text { arallo: }}$ a new $M_{\text {Like }}$ alpha 1-antitrypsin-deficient allele. Diagn Mol Pathol 2003;12:237-9.

25. Fra AM, Gooptu B, Ferrarotti I, Miranda E, Scabini R, Ronzoni R, et al. Three new alpha1-antitrypsin deficiency variants help to define a C-terminal region regulating conformational stability and polymerization. PLoS One 2012;7:e38405.

26. Miravitlles M, Dirksen A, Ferrarotti I, Koblizek V, Lange P, Mahadeva R, et al. European Respiratory Society statement: diagnosis and treatment of pulmonary disease in $\alpha 1$-antitrypsin deficiency. Eur Respir J 2017;50:1700610.

27. Zorzetto M, Russi E, Senn O, Imboden M, Ferrarotti I, Tinelli C, et al. SERPINA1 gene variants in individuals from the general population with reduced alpha1-antitrypsin concentrations. Clin Chem 2008;54:1331-8. 
28. Senn O, Russi EW, Schindler C, Imboden M, von Eckardestein A, Bradli 0 , et al. Circulating alpha1-antitrypsin in the general population: determinants and association with lung function. Respir Res 2008;9:35.

29. Sanders CL, Ponte A, Kueppers F. The effects of inflammation on alpha 1 antitrypsin levels in a national screening cohort. COPD 2018;15:10-16.

30. Ferrarotti I, Baccheschi J, Zorzetto M, Tinelli C, Coni P, Corda L, et al. Prevalence of rare variants in the Italian registry for severe alpha 1-antitrypsin deficency. J Med Genet 2005;42:282-7.

31. Silva D, Oliveira MJ, Guimarães M, Lima R, Gomes S, Janciauskiene $\mathrm{S}$, et al. Alpha-1-antitrypsin (SERPINA1) mutation spectrum: three novel variants and haplotype characterization of rare deficiency alleles identified in Portugal. Respir Med 2016; 116:8-18.

32. Jameson JL, Longo DL. Precision medicine-personalized, problematic, and promising. N Engl J Med 2015;372: 2229-34.

33. Brantly M, Campos M, Davis AM, D’Armiento J, Goodman K, Hanna $\mathrm{K}$, et al. Detection of alpha-1 antitrypsin deficiency: the past, present and future. Orphanet J Rare Dis 2020;15:96.

34. Gainotti S, Mascalzoni D, Bros-Facer V, Petrini C, Floridia G, Roos M, et al. Meeting patients' right to the correct diagnosis: ongoing international initiatives on undiagnosed rare diseases and ethical and social issues. Int J Environ Res Public Health 2018;15:2072. 